\title{
diffusion-înolarrentals
}

The Open-Access Journal for the Basic Principles of Diffusion Theory, Experiment and Application

\section{Molecular Traffic Control in Porous Nanoparticles}

\author{
Andreas Brzank ${ }^{1,2}$, Gunter Schütz ${ }^{2}$ \\ ${ }^{1}$ Fakultät für Physik und Geowissenschaften, Universität Leipzig, Abteilung \\ Grenzflächenphysik, Linnestrasse 5, D-04103, Germany \\ ${ }^{2}$ Institut für Festkörperforschung, Forschungszentrum Jülich, D-52425 Jülich, Germany
}

Corresponding author:

Andreas Brzank

E-Mail: brzank@uni-leipzig.de

\begin{abstract}
We investigate the conditions for reactivity enhancement of catalytic processes in porous solids by use of molecular traffic control (MTC) as a function of reaction rate and grain size. With dynamic Monte-Carlo simulations, continuous-time random walk theory and solutions of the Master equation we obtain a quantitative description of the MTC effect for a network of intersecting single-file channels in a wide range of grain parameters.
\end{abstract}

Keywords: molecular traffic control, discrete poisson equation, random walk, single file, symmetric exclusion

\section{Introduction}

Zeolites are used for catalytic processes in a variety of applications, e.g. cracking of large hydrocarbon molecules. In a number of zeolites diffusive transport occurs along quasione-dimensional channels which do not allow guest molecules to pass each other (singlefile behaviour). Hence, the effective reactivity, determined by the residence time of molecules in the zeolite, may be considerably reduced as compared to the reactivity in the absence of single-file behaviour. It has been suggested that the single-file effect may be circumvented by the so far controversial concept of molecular traffic control (MTC) $[1,2]$. This notion rests on the assumption that reactant and product molecules resp. may prefer spatially separated diffusion pathways and thus avoid mutual suppression of selfdiffusion inside the grain channels. Whether an MTC effect leading to reactivity enhancement actually takes place was addressed in a series of dynamic Monte Carlo simulations (DMCS) of a stochastic model system with a network of perpendicular sets of bimodal intersecting channels and with catalytic sites located at the intersecting pores (NBK model) $[3,4]$. The occurrence of an MTC effect was found by comparing the outflow of reaction products in the MTC system with the outflow from a reference system with equal internal and external system parameters, but no channel selectivity.

\section{Numeration of results}

The efficiency ratio of MTC systems with catalytic conversion $A \rightarrow B$ (compared with a topologically and structurally similar reference system without MTC and optimal external conditions) is inversely proportional to the grain diameter. The MTC effect is favored by 
a small number of channels and long channel segments between intersections, which by themselves lead to a very low absolute outflow [3,4,5]. An analytical treatment of the master equation for this stochastic many-particle model revealed the origin of this effect at high reactivities [6]. It results from an interplay of long residence time of guest molecules under single-file conditions with a saturation effect that leads to a depletion of the bulk of the crystallite and we find the output of B-particles being independent of the number of channels. The MTC effect decreases strongly with increasing reactivity $c$ above some optimal value $c^{*}$. Nevertheless, there is an MTC effect for any number of channels and any distance between reactive sites. For small grains MTC leads to a reactivity enhancement of up to approximately 30\% [5]. Using a similar strategy we solve the case for small reactivities and find a very good coincidence with our simulations [5]. In the low concentration regime we describe the diffusion for the reference system with a random walk ansatz leading to an effective diffusion equation [5]. The situation in 3D is similar to the case in two dimensions. Therefore an MTC effect is expected for systems with small number of channels corresponding to porous nanoparticles [7].

\section{Conclusion and Outlook}

In summary, our investigations suggest that MTC may enhance significantly the effective reactivity in zeolitic nanoparticles with suitable binary channel systems and thus may be of practical relevance in applications. There has been intense research activity in the design and synthesis of structured mesoporous solids with a controlled pore size. In particular, synthesis of bimodal nanostructures with independently controlled small and large mesopore sizes has become feasible [10]. The necessary (but not sufficient) requirement for the MTC effect, a channel selectivity of two different species of molecules, has been verified by means of molecular dynamic (MD) simulations $[8,9]$. This makes further investigation of the MTC effect promising. In particular we intend to study new channel topologies in order to overcome the negative $N$ dependence, with total selectivity as well as size-selectivitiy where the smaller B particles are allowed to enter both $\alpha$ and $\beta$ channels. Our preliminary results open new perspectives which are not considered in the first period. Other important issues are: a) Finding finite size corrections for systems with short file length. b) The study of system rates.

\section{References}

[1] E.G. Derouane and Z. Gabelica, J. Catal. 65 (1980) 486.

[2] E.G. Derouane, Appl. Catal. A, N2 (1994) 115.

[3] N. Neugebauer, P. Bräuer, J. Kärger, J. Catal. 194 (2000) 1.

[4] P. Bräuer, A. Brzank,J. Kärger, J. Phys. Chem. B 107 (2003) 1821.

[5] A. Brzank, G. M. Schütz, Appl. Catal. A to appear

[6] A. Brzank, G. M. Schütz, P. Bräuer, and J. Kärger, Phys. Rev. E 69 (2004) 031102.

[7] A. Brzank,S. Kwon,G. M. Schütz, Diffusion Fundamentals submitted

[8] R. Q. Snurr and J. Kärger, Phys. Chem. B 101 (1997) 6469.

[9] L. A. Clark,G. T. Ye, R. Q. Snurr, Phys. Rev. Lett. 84 (2000) 2893.

[10] J.H. Sun, Z. Shan, Th. Maschmeyer, M.-O. Coppens, Langmuir 19 (2003) 8395. 